\title{
Empathy: The Role of Expectations
}

\author{
Sabrina Trapp \\ Gonda Multidisciplinary Brain Research Center, Bar-Ilan University, Israel \\ Department of Psychology, Ludwig-Maximilians-University, Germany \\ Simone Schütz-Bosbach \\ Department of Psychology, Ludwig-Maximilians-University, Germany \\ Moshe Bar \\ Gonda Multidisciplinary Brain Research Center, Bar-Ilan University, Israel
}

\begin{abstract}
To what extent can we feel what someone else feels? Data from neuroscience suggest that empathy is supported by a simulation process, namely the neural activation of the same or similar regions that subserve the representation of specific states in the observer. However, expectations significantly modulate sensory input, including affective information. For example, expecting painful stimulation can decrease the neural signal and the subjective experience thereof. For an accurate representation of the other person's state, such top-down processes would have to be simulated as well. However, this is only partly possible, because expectations are usually acquired by learning. Therefore, it is important to be aware of possible misleading simulations that lead to misinterpretations of someone's state.
\end{abstract}

\section{Keywords}

affect sharing, emotional contagion, mirror neurons, modulation, top-down

\section{Neuroscientific Study of Empathy}

To what extent can we feel what someone else feels? In a famous thought experiment, Wittgenstein (1953) compared the subjective experience of pain to a beetle in a box: We all have this beetle in a box, but no one can actually look into anyone else's box; we can only use words to refer to the content in the box. While Wittgenstein's analogy was not intended to describe empathy, it draws attention to possible obstacles towards sharing and understanding someone's internal states. What does neuroscience have to offer to address this issue?

Although the ability to empathize with a conspecific's affective state has been reported in animals, it is often considered as one of the core processes that make us human. Therefore, it is no surprise that researchers have been trying to unravel the underlying mechanisms that subserve this ability. Several researchers consider empathy as a concept that entails two facets. The first is mentalizing - this refers to the ability to cognitively represent someone's perspective (Zaki \& Ochsner, 2012). This process has been more closely associated with the concept of theory of mind, which refers to the ability to attribute mental states (such as desires, beliefs, intentions, and knowledge) to another mind (Premack \& Woodruff, 1978). In laboratory settings, this is usually investigated by exposing participants to videos, scenes, written or spoken text material that is either emotional or neutral for a protagonist. The ability to mentalize is assessed by asking participants to predict a possible reaction or to infer the mental state of the protagonist. The second facet, affect sharing, refers to a process by which someone's internal state is actually experienced by the observer, that is, having a state "isomorphic to

Author note: We thank Laura Levine for proofreading the manuscript. This study was supported by the Israeli Center of Research Excellence in Cognition (I-CORE) Grant $51 / 11$ (MB). 
another person's affective state" (De Vignemont \& Singer, 2006, p. 435).

In the past decade, empathic processes have often been explained with shared neural activation. That is, I can empathize because observing another person's state activates the regions in my brain that represent or process such state. The roots historically date back to a single-cell recording study by di Pellegrino, Fadiga, Fogassi, Gallese, and Rizzolatti (1992), who demonstrated that the inferior premotor cortex in the monkey, which is involved in holding and grasping movements, was also active when the monkey merely observed these actions in the experimenter. Therefore, the phenomenon has been termed "mirror neurons," and similar effects have been shown in humans with imaging methods a few years later (Rizzolatti, Fadiga, Gallese, $\&$ Fogassi, 1996). If action understanding may be enabled by a simulation, what about understanding or sharing someone's sensory, affective, or bodily states, that is, being able to empathize? Although neuronal mirror activity in humans awaits empirical support and its functional role for empathy requires conceptual clarification, there is evidence that merely observing someone during a specific stimulation elicits neural processes in the observer that are also involved in the actual experience of this stimulation. For example, using functional magentic resonance imaging (fMRI), Singer et al. (2004) showed that the neural underpinnings of pain observation resembled those that are associated with experiencing pain. These findings were supported with multivariate pattern analysis techniques which revealed the same local activation pattern when participants observed and experienced pain (Corradi-Dell'Acqua, Hofstetter, \& Vuilleumier, 2011). Likewise, the experience of touch has been shown to activate the somatosensory cortex, regardless of whether participants merely watched a video showing another person being touched, or whether they were actually touched while being scanned (e.g., Keysers et al., 2004). More recent evidence suggests that this effect can even be found in the primary somatosensory cortex (Kuehn, Mueller, Turner, \& SchützBosbach, 2014). Wicker et al. (2003) demonstrated that the anterior insula, a region involved in processing disgust, was also activated when participants just watched a video clip showing a person expressing disgust. Furthermore, neural regions associated with pleasant versus unpleasant stimulation were also recruited when such events were merely observed (Lamm, Silani, \& Singer, 2015).

However, explaining action understanding and empathy with shared neural activity has been criticized on several grounds (e.g., Lamm \& Majdandžić, 2015; Zaki \& Ochsner, 2012). For example, an overlap of fMRI activity does not necessarily imply the recruitment of the same populations of neurons (Logothetis, 2008). Direct evidence would come from disruptions or lesions of respective areas that result in a deficit in the ability to empathize. Although there are some studies that have addressed the issue of causality (e.g., Leigh et al., 2013; Steinbeis, Bernhardt, \& Singer, 2015), further investigations are warranted. SchützBosbach and Prinz (2007) suggested that our sensitivity to perceive specific actions increases for those actions that we ourselves have performed ("perceptual resonance"). This implies that our action understanding may be limited to our own motor expertise. What are the limitations of sharing someone's affects? Here, we aim to draw attention to the role of expectations for the simulation of someone else's sensory and affective states.

\section{Expectations: A Key to Understanding the Brain}

Our perceptual system shows an amazing efficiency in identifying sensory input, despite noise, ambiguity, and considerable variations in the way it is presented. The motor system, too, is exceptionally smooth in navigating us through a world full of objects and possible obstacles, even with significant delays of sensory input due to receptor and neural conduction time. The artificial simulation of complex perception and action still poses a major challenge to robotics. How does the brain solve this computational problem? In cognitive neuroscience, there is broad consensus that a main avenue towards understanding brain function is to unravel the mechanisms of predictions (Bar, 2007; Friston, 2010; Rao \& Ballard, 1999). For example, predicting how the arm moves in response to a motor command can counteract delays in sensory input (Kording \& Wolpert, 2004; Wolpert \& Flanagan, 2001). Furthermore, prior information can disambiguate sensory input and fill in where noise and clutter prevent unequivocal interpretation (Summerfield \& de Lange, 2014; Trapp \& Bar, 2015).

The influence of expectations on sensory input is referred to as a top-down process. In cognitive neuroscience, the term usually implies a modulation of bottom-up input (Gilbert \& $\mathrm{Li}$, 2013). Depending on the definition, top-down modulation refers to explicit goals or hypotheses about sensory inputs, anatomical descending connections that can modulate bottom-up information, or to the local entrainment of neuronal populations by distant brain regions (for a discussion see Rauss \& Pourtois, 2013). The source of such top-down modulations may be attention (Hopfinger, Buonocore, \& Mangun, 2000), reward history (Awh, Belopolsky, \& Theeuwes, 2012), or expectations (Bar, 2003). The focus here is on the role of expectations. ${ }^{1}$

A major index for the influence of expectations on sensory processing is a reduced neural signal for predicted, and an increased signal for unpredicted input. Both neural decreases and increases have been reported in different modalities and with various methods, such as encephalography, magnetoencephalography, and fMRI (e.g., Alink, Schwiedrzik, Kohler, Singer, \& Muckli, 2010; Bendixen, Schwartze, \& Kotz, 2015; Brodski, Paasch, Helbling, \& Wibral, 2015; Kimura, Kondo, Ohira, \& Schröger, 2012; Kok, Failing, \& de Lange, 2014; Todorovic, van Ede, Maris, \& de Lange, 2011; Turk-Browne, Scholl, Johnson, \& Chun, 2010). Such expectation effects, together with the highly feedback-dependent architecture of the brain, fueled the formulation of theoretical frameworks like the Bayesian brain and predictive coding (Friston, 2010; Knill \& Pouget, 2004; Rao \& Ballard, 1999). The key idea in predictive coding is that deviations of expectations, the prediction errors, help to iteratively refine the generative model that explains the input. 
How do expectations modulate affective processing? Researchers in the early days of behavioral neuroscience tested whether the anticipation of an electrical shock in rats led to a significant alteration of behavior that is linked to the experience of stress. Indeed, when the aversive stimulation could be anticipated, the rats expressed less muscle tension and ulceration (Mineka \& Kihlstrom, 1978; Seligman, 1968; Weiss, 1970). If human participants were exposed to painful stimuli that were unexpected, the activation in the network associated with pain processing was stronger (Seidel et al., 2015). Expectations can also enhance sensory processing: For example, when pain is expected, other, nonpainful tactile stimuli are more easily detected, indicating a higher general excitability (van Hulle, Durnez, Crombez, \& van Damme, 2015). In a study by Keltner et al. (2006), participants reported significantly more pain when a highly noxious thermal stimulus was applied and a high pain intensity had been expected. Importantly, the expectations also enhanced the activation of the afferent pain circuitry at the thalamic and cortical level. Porro et al. (2002) applied painful subcutaneous injection of ascorbic acid and found that fMRI activity increased during the anticipation of this stimulation in the somatosensory cortex. Such enhanced neural responses have also been reported when participants had actually expected pain, but received nonpainful somatosensory stimulation (Sawamoto et al., 2000).

There are various factors that play a modulatory role in the interplay between pain expectation and pain processing (Atlas \& Wager, 2012; Ploghaus, Becerra, Borras, \& Borsook, 2003). Recently, de Berker et al. (2016) found evidence that the increased pain experience may be primarily due to uncertainty in conditions of unexpected stimulation. In their study, participants played a computer game while their stress level was measured by tracking several physiological parameters, such as salivary cortisol, skin conductance, and pupil diameter. During the game, participants were exposed to mild electrical shocks that were either $100 \%, 50 \%$, or $0 \%$ predictable. The authors found that the stress level was highest when the occurrence probability of the shock was at chance level (i.e., 50\% predictability). In this condition, the response was even greater than the reaction to pain that could not be anticipated at all $(0 \%$ predictability).

\section{Empathy, Top-Down Processes, and Expectations}

Top-down processes have also been shown to play a role in empathic processes, and modulate responses accordingly. For example, when observing painful medical treatments, neural evaluation systems like the orbitofrontal cortex showed increased activity when the observers learned that the treatment was not successful for the observed person (Lamm, Batson, \& Decety, 2007). This study therefore demonstrates that empathic responses can be shaped and regulated (by post hoc knowledge in this case), and are not only determined by what the observed person felt. In a study by Lamm, Nusbaum, Meltzoff, and Decety (2007), participants saw photographs of hands that displayed painful needle injections. In one condition, observers were informed that the injection was performed on an anesthetized hand. In this case, the medial and lateral parts of the prefrontal cortex were activated to a higher extent as compared to viewing photographs with a nonnumb hand. These regions have been linked to various modulatory processes, such as emotion regulation and general evaluation, and in this context may indicate downregulation of empathic responses. This interpretation is supported by region-of-interest analyses in the same study that revealed less activation in the right anterior insula during the observation of treatments of numb hands. The insula is considered to be an important area for the processing of pain. The reduced activity may be a result of a top-down attenuation of the empathic response. Pehrs et al. (2017) investigated the role of the temporal pole, a region that has previously been linked to the integration of contextual knowledge. When participants observed emotional film clips, the connectivity between the temporal pole and the fusiform gyrus increased when contextual knowledge was incorporated. The fusiform gyrus is part of the ventral visual stream, and processes perceptual information. The increased connectivity therefore indicates a topdown modulation of this area when additional information was incorporated.

In the experiments reported before, contextual information was provided by instruction, that is, informing participants about the circumstances of the observed person. This would in principle allow regulating the strength of one's own empathic response, and to accurately simulate the stimulation applied to another person. But the influence of modulatory information and top-down processes is not limited to cases in which topdown influence or knowledge is explicit.

Expectations are often the result of acquiring covariations and statistical regularities of our sensory environment over time. In the laboratory, there are several ways to investigate such processes under controlled conditions. For example, in a visual search paradigm, called contextual cueing, participants search through seemingly random layouts for a specific target whose identity remains the same throughout the experiment. Unbeknownst to them, some "predictable" layouts are repeated several times during the experiment, and here participants learn to find the position of the target faster. This behavioral advantage is interpreted as reflecting the ability of humans to exploit spatial contextual regularities (Chun \& Jiang, 1998). Serial stimulation with embedded visual or acoustic regularities (i.e., event B follows event A) enables anticipation of upcoming input, and it has been shown that the neural response to predicted stimulation decreases (e.g., Turk-Browne et al., 2010). Such behavioral benefits and reduced neural signals to predicted events would not occur if someone did not undergo the same learning history. Furthermore, perceptual events are usually contextually associated with other events; for example, a knife may trigger the image of a fork and may therefore foster a prediction (Bar, 2007). While we presumably share a considerable amount of such semantic associations, each individual's history may render some associations highly idiosyncratic. For example, one person may 
learn to expect tulips on a kitchen table, whereas for someone else, this would elicit strong emotions (e.g., happiness and surprise). An accurate simulation of the emotional reaction by an observer would require incorporating such learned expectations. The role of expectations in the context of empathy is illustrated in Figure 1.

A repeated exposure to a stimulus can also elicit expectations. Repetition suppression (RS) is a phenomenon that refers to a reduced neural signal when a stimulus is repeated (for a review see Grill-Spector, Henson, \& Martin, 2006). In the context of predictive coding, this effect has been interpreted as a reduced prediction error, assuming that the continuous exposure evokes expectations of the stimulus (Auksztulewicz \& Friston, 2016). First evidence for this idea was provided by Summerfield, Trittschuh, Monti, Mesulam, and Egner (2008) who found that the RS effect was modulated by the likelihood of a repetition, which would not occur if the effect is entirely driven by (lowlevel) neural fatigue or adaptation effects. There is evidence that RS also changes as a function of the stimulus' valence (for a review see Trapp \& Kotz, 2016). Expectation effects of RS are usually automatic and participants report no awareness of repetition likelihoods (Grotheer \& Kovács, 2016). In order to simulate the influence of repetitions, the observing person would have to undergo the same repetitive exposure or likelihood manipulation.

In the context of implicitly acquired expectations, age may play an important role as well. Intuitively, the longer we live, the better we learn the structure of our environment. This may lead to greater reliance on top-down processes. Support for this idea comes from a study which fitted three different connectivity models to data from an object naming task —included brain areas were the early visual cortex, anterior ventral temporal cortex, angular gyrus, and inferior frontal gyrus (Gilbert \& Moran, 2016). Data from aged individuals showed a better fit with models that incorporated predictive coding principles. Furthermore, frontal activity occurred earlier and was higher in age. Moreover, there is evidence that model updating, a process that recalibrates current internal models of the environment, is attenuated in aged brains, suggesting less sensitivity to bottom-up signals (Moran, Symmonds, Dolan, \& Friston, 2014). This may be important in the context of empathizing with aged individuals.

\section{Clinical Implications}

A lack of empathy not only increases suffering of people that need social consolation in highly aversive situations, it also renders social interactions difficult for individuals that have deficits in empathic abilities. Therefore, tools that allow to accurately diagnose empathic response skills are of high value. We here outlined that an important facet of empathy is the ability to incorporate the modulatory influence of expectations. Expectations are to a high degree the result of an individual learning history, and can therefore not exhaustively be simulated by an external observer. Nevertheless, some expectations can be explicitly triggered. There is evidence for neural net-

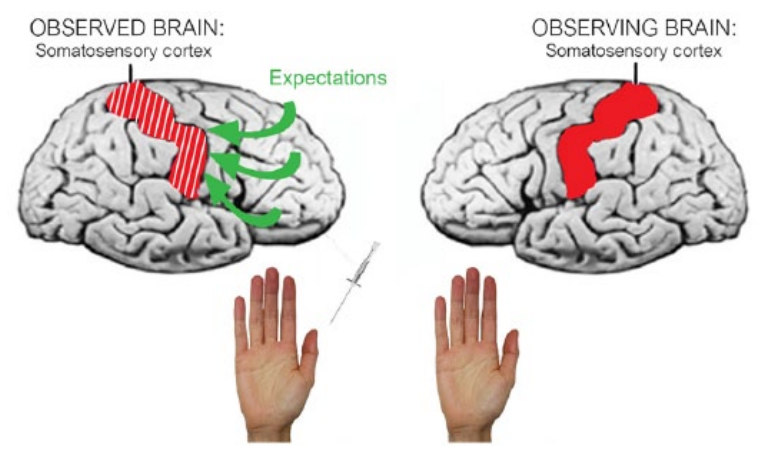

Figure 1. Empathy and expectations. Expectations modulate processing of sensory input. Left: Observed person. The somatosensory cortex is activated by stimulation of the hand by a needle, but the neural response (and subjective experience) is attenuated by the expectation hereof. Right: Observing person. The somatosensory cortex is activated by observing the stimulation of the other person, but the neural response is not attenuated, because the expectation of the stimulation is not incorporated.

works that specifically subserve explicit expectation of emotional events. Bermpohl et al. (2006) used images depicting various emotional scenes, and either announced the valence of the upcoming image (neutral or emotional) or presented the image without any preceding information; the valence of the emotion was not announced. The authors found that emotional expectancy specifically produced activation in regions not associated with emotion perception, and excluded arousal as a confounding factor. Such regions may be important to look at during clinical assessments to understand whether and which facet of empathy is disturbed in an observer, that is, is it the simulation of actual sensations, or their modulation by expectations. Paradigms that use cues that announce an event may be particularly well suited. For example, patients with deficits in empathy could be asked to observe another person who receives painful treatments that are announced by a cue (i.e., expected) or not. Imaging methods can be used to unravel neural activity in both representational and modulatory brain areas of the observer. Empathic dysfunctions in disorders such as autism may be explained by deficits in top-down processing. This aligns nicely with a recent proposal that key symptoms of autistic individuals are linked to perceiving the world with less expectations (Pellicano \& Burr, 2012).

\section{Summary}

Intuitively, we know that it is not possible to exactly feel what someone else feels. We here provided an explanation from a neuroscientific point of view, and outlined that in order to accurately share someone's state, not only the state has to be represented, but its underlying top-down dynamics have to be incorporated as well. Top-down contributions based on expectations are only partly - if at all — accessible by mere observation. Certainly, empathy does not require an observer to feel exactly what someone else feels. For example, 
someone is sad because she had just received bad news, but her sorrow is modulated by the fact that she was expecting the news. The observer who empathizes with this sadness will not be experiencing the same "tone" or degree of sorrow, but will still be able to empathize. But the intensity of the observer's simulated experience remains unchanged and strong. Due to the fact that the other person's expectations defy a direct observation, misleadingly strong empathic responses are possible.

\section{Declaration of Conflicting Interests}

The author(s) declared no potential conflicts of interest with respect to the research, authorship, and/or publication of this article.

\section{Note}

1 While most studies investigated the expectation of the identity of a stimulus (expecting what), there is evidence that manipulating the onset of an event (expecting when/temporal attention) results in similar neural modulations and behavioral benefits (Correa \& Nobre, 2008; Doherty, Rao, Mesulam, \& Nobre, 2005). Therefore, further differentiation between these two kinds of expectations is not discussed.

\section{References}

Alink, A., Schwiedrzik, C. M., Kohler, A., Singer, W., \& Muckli, L. (2010). Stimulus predictability reduces responses in primary visual cortex. Journal of Neuroscience, 30(8), 2960-2966.

Atlas, L. Y., \& Wager, T. D. (2012). How expectations shape pain. Neuroscience Letters, 520(2), 140-148.

Auksztulewicz, R., \& Friston, K. (2016). Repetition suppression and its contextual determinants in predictive coding. Cortex, 80, 125-140.

Awh, E., Belopolsky, A. V., \& Theeuwes, J. (2012). Top-down versus bottom-up attentional control: A failed theoretical dichotomy. Trends in Cognitive Sciences, 16(8), 437-447.

Bar, M. (2003). A cortical mechanism for triggering top-down facilitation in visual object recognition. Journal of Cognitive Neuroscience, 15, 600-609.

Bar, M. (2007). The proactive brain: Using analogies and associations to generate predictions. Trends in Cognitive Sciences, 11(7), 280-289.

Bendixen, A., Schwartze, M., \& Kotz, S. A. (2015). Temporal dynamics of contingency extraction from tonal and verbal auditory sequences. Brain and Language, 148, 64-73.

Bermpohl, F., Pascual-Leone, A., Amedi, A., Merabet, L. B., Fregni, F., Gaab, N., . . Northoff, G. (2006). Dissociable networks for the expectancy and perception of emotional stimuli in the human brain. Neuroimage, 30, 588-600.

Brodski, A., Paasch, G. F., Helbling, S., \& Wibral, M. (2015). The faces of predictive coding. Journal of Neuroscience, 35(24) 8997-9006.

Chun, M. M., \& Jiang, Y. (1998). Contextual cueing: Implicit learning and memory of visual context guides spatial attention. Cognitive Psychology, 36(1), 28-71.

Corradi-Dell'Acqua, C., Hofstetter, C. P., \& Vuilleumier, P. (2011). Felt and seen pain evoke the same local patterns of cortical activity in insular and cingulate cortex. The Journal of Neuroscience, 31, 1799618006.

Correa, A., \& Nobre, A. C. (2008). Neural modulation by regularity and passage of time. Journal of Neurophysiology, 100, 1649-1655.

De Berker, A. O., Rutledge, R. B., Mathys, C., Marshall, L., Cross, G. F., Dolan, R. J., \& Bestmann, S. (2016). Computations of uncertainty mediate acute stress responses in humans. Nature Communications, 7 , 10996. doi: $10.1038 /$ ncomms 10996

De Vignemont, F., \& Singer, T. (2006). The empathic brain: How, when and why? Trends in Cognitive Sciences, 10, 435-441.

Di Pellegrino, G., Fadiga, L., Fogassi, L., Gallese, V., \& Rizzolatti, G. (1992). Understanding motor events: A neurophysiological study. Experimental Brain Research, 91(1), 176-180.

Doherty, J. R., Rao, A., Mesulam, M. M., \& Nobre, A. C. (2005). Synergistic effect of combined temporal and spatial expectations on visual attention. Journal of Neuroscience, 25, 8259-8266.

Friston, K. (2010). The free-energy principle: A unified brain theory? Nature Reviews: Neuroscience, 11, 127-138.

Gilbert, C. D., \& Li, W. (2013). Top-down influences on visual processing. Nature Reviews: Neuroscience, 14, 350-363.

Gilbert, J. R., \& Moran, R. J. (2016). Inputs to prefrontal cortex support visual recognition in the aging brain. Scientific Reports, 6, 31943. doi: $10.1038 /$ srep31943

Grill-Spector, K., Henson, R., \& Martin, A. (2006). Repetition and the brain: Neural models of stimulus-specific effects. Trends in Cognitive Science, 10(1), 14-23.

Grotheer, M., \& Kovács, G. (2016). Can predictive coding explain repetition suppression? Cortex, 80, 113-124.

Hopfinger, J. B., Buonocore, M. H., \& Mangun, G. R. (2000). The neural mechanisms of top-down attentional control. Nature Neuroscience, 3 , 284-291.

Keltner, J. R., Furst, A., Fan, C., Redfern, R., Inglis, B., \& Fields, H. L. (2006). Isolating the modulatory effect of expectation on pain transmission: A functional magnetic resonance imaging study. The Journal of Neuroscience, 26(16), 4437-4443.

Keysers, C., Wicker, B., Gazzola, B., Anton, J. L., Fogassi, L., \& Gallese, V. (2004). A touching sight: SII/PV activation during the observation and experience of touch. Neuron, 42, 335-346.

Kimura, M., Kondo, H., Ohira, H., \& Schröger, E. (2012). Unintentional temporal context-based prediction of emotional faces: An electrophysiological study. Cerebral Cortex, 22(8), 1774-1785.

Knill, D. C., \& Pouget, A. (2004). The Bayesian brain: The role of uncertainty in neural coding and computation. Trends in Neurosciences, 27(12), 712-719.

Kok, P., Failing, M., \& de Lange, F. P. (2014). Prior expectations evoke stimulus templates in the primary visual cortex. Journal of Cognitive Neuroscience, 26, 1546-1554.

Kording, K. P., \& Wolpert, D. M. (2004). Bayesian integration in sensorimotor learning. Nature, 427, 244-247.

Kuehn, E., Mueller, K., Turner, R., \& Schütz-Bosbach, S. (2014). The functional architecture of S1 during touch observation described with $7 \mathrm{~T}$ fMRI. Brain Structure and Function, 219(1), 119-140.

Lamm, C., Batson, C. D., \& Decety, J. (2007). The neural substrate of human empathy: Effects of perspective-taking and cognitive appraisal. Journal of Cognitive Neuroscience, 19, 42-58.

Lamm, C., \& Majdandžić, J. (2015). The role of shared neural activations, mirror neurons, and morality in empathy - A critical comment. Neuroscience Research, 90, 15-24.

Lamm, C., Nusbaum, H. C., Meltzoff, A. N., \& Decety, J. (2007). What are you feeling? Using functional magnetic resonance imaging to assess the modulation of sensory and affective responses during empathy for pain. PLoS One, 2(12), e1292. doi:10.1371/journal.pone.0001292

Lamm, C., Silani, G., \& Singer, T. (2015). Distinct neural networks underlying empathy for pleasant and unpleasant touch. Cortex, 70, 79-89.

Leigh, R., Oishi, K., Hsu, J., Lindquist, M., Gottesman, R. F., Jarso, S., . . Hillis, A. E. (2013). Acute lesions that impair affective empathy. Brain, $136,2539-2549$.

Logothetis, N. K. (2008). What we can do and what we cannot do with fMRI. Nature, 453, 869-878. 
Mineka, S., \& Kihlstrom, J. F. (1978). Unpredictable and uncontrollable events: A new perspective on experimental neurosis. Journal of Abnormal Psychology, 87(2), 256-271.

Moran, R. J., Symmonds, M., Dolan, R. J., \& Friston, K. J. (2014). The brain ages optimally to model its environment: Evidence from sensory learning over the adult lifespan. PLoS Computational Biology, 10, e1003422.

Pehrs, C., Zaki, J., Schlochtermeier, L. H., Jacobs, A. M., Kuchinke, L., \& Koelsch, S. (2017). Temporal pole top-down modulates the ventral visual stream during social cognition. Cerebral Cortex, 27(1), 777-792.

Pellicano, E., \& Burr, D. (2012). When the world becomes "too real": A Bayesian explanation of autistic perception. Trends in Cognitive Sciences, 16, 504-510.

Ploghaus, A., Becerra, L., Borras, C., \& Borsook, D. (2003). Neural circuitry underlying pain modulation: Expectation, hypnosis, placebo. Trends in Cognitive Sciences, 7, 197-200.

Porro, A. C., Baraldi, P., Pagnoni, G., Serafini, M., Facchin, P., Maieron, M., \& Nichelli, P. (2002). Does anticipation of pain affect cortical nociceptive systems? The Journal of Neuroscience, 22(8), 3206-3214.

Premack, D., \& Woodruff, G. (1978). Does the chimpanzee have a theory of mind? Behavioral Brain Science, 1(4), 515-526.

Rao, R. P., \& Ballard, D. H. (1999). Predictive coding in the visual cortex: A functional interpretation of some extra-classical receptive-field effects. Nature Neuroscience, 2(1), 79-87.

Rauss, K., \& Pourtois, G. (2013). What is the bottom-up and what is the top-down in predictive coding? Frontiers in Psychology, 4(276), 1-8.

Rizzolatti, G., Fadiga, L., Gallese, V., \& Fogassi, L. (1996). Premotor cortex and the recognition of motor actions. Brain Research: Cognitive Brain Research, 3, 131-141.

Sawamoto, N., Honda, M., Okada, T., Hanakawa, T., Kanda, M., Fukuyama, H., . . . Shibasaki, H. (2000). Expectation of pain enhances responses to nonpainful somatosensory stimulation in the anterior cingulate cortex and parietal operculum/posterior insula: An event-related functional magnetic resonance imaging study. Journal of Neuroscience, 20, 7438-7445.

Schütz-Bosbach, S., \& Prinz, W. (2007). Perceptual resonance: Actioninduced modulation of perception. Trends in Cognitive Sciences, 11(8), 349-355.

Seidel, E. M., Pfabigan, D. M., Hahn, A., Sladky, R., Grahl, A., Paul, K., . . . Lamm, C. (2015). Uncertainty during pain anticipation: The adaptive value of preparatory processes. Human Brain Mapping, 36(2), 744-755.
Seligman, M. E. P. (1968). Chronic fear produced by unpredictable electric shock. Journal of Comparative and Physiological Psychology, 66, 402-411.

Singer, T., Seymour, B., O’Doherty, J., Kaube, H., Dolan, R. J., \& Frith, C. D. (2004). Empathy for pain involves the affective but not sensory components of pain. Science, 303, 1157-1162.

Steinbeis, N., Bernhardt, B. C., \& Singer, T. (2015). Age-related differences in function and structure of rSMG and reduced functional connectivity with DLPFC explains heightened emotional egocentricity bias in childhood. Social Cognitive and Affective Neuroscience, 10(2), 302-310.

Summerfield, C., \& de Lange, F. P. (2014). Expectation in perceptual decision-making: Neural and computational mechanisms. Nature Reviews: Neuroscience, 15, 745-756.

Summerfield, C., Trittschuh, E. H., Monti, J. M., Mesulam, M. M., \& Egner, T. (2008). Neural repetition suppression reflects fulfilled perceptual expectations. Nature Neuroscience, 11(9), 1004-1006.

Todorovic, A., van Ede, F., Maris, E., \& de Lange, F. P. (2011). Prior expectation mediates neural adaptation to repeated sounds in the auditory cortex: An MEG study. Journal of Neuroscience, 31(25), 9118-9123.

Trapp, S., \& Bar, M. (2015). Prediction, context and competition in visual recognition. Annals of the New York Academy of Sciences, 339, 190-198.

Trapp, S., \& Kotz, S. (2016). Affective prediction - An evaluation of repetition suppression effects. Frontiers in Psychology, 7, 1365. doi:10.3389/ fpsyg.2016.01365

Turk-Browne, N. B., Scholl, B. J., Johnson, M. K., \& Chun, M. M. (2010). Implicit perceptual anticipation triggered by statistical learning. Journal of Neuroscience, 30, 11177-11187.

Van Hulle, L., Durnez, W., Crombez, G., \& van Damme, S. (2015). Detection of tactile change detection on a bodily location where pain is expected. Perceptual and Motor Skills, 120(1), 219-231.

Weiss, J. M. (1970). Somatic effects of predictable and unpredictable shock. Psychosomatic Medicine, 77, 14-21.

Wicker, B., Keysers, C., Plailly, J., Royet, J. P., Gallese, V., \& Rizzolatti, G. (2003). Both of us disgusted in My insula: The common neural basis of seeing and feeling disgust. Neuron, 40, 655-664.

Wittgenstein, L. (1953). Philosophical investigations. Oxford, UK: Basil Blackwell.

Wolpert, D. M., \& Flanagan, J. R. (2001). Motor prediction. Current Biology, 18, 729-732.

Zaki, J., \& Ochsner, K. (2012). The neuroscience of empathy: Progress, pitfalls, and promise. Nature Neuroscience, 15(5), 675-680. 